\title{
Research And Implementation For Remote Access Of UPnP
}

\author{
Gan Xiaojian ${ }^{1,}$, Wang Haitao ${ }^{2,}$ b, Jiang Ying ${ }^{3, c}$, Chen Xing ${ }^{4}$ \\ ${ }^{1}$ Kunming University of Science and Technology, Yunnan Province, Kunming City \\ ${ }^{2}$ Kunming University of Science and Technology, Yunnan Province, Kunming City \\ ${ }^{2}$ Kunming University of Science and Technology, Yunnan Province, Kunming City \\ ${ }^{2}$ Kunming University of Science and Technology, Yunnan Province, Kunming City

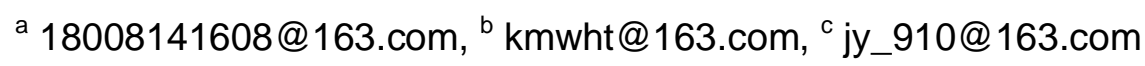

Keywords: UPnP, Intelligent terminal, Web Server, Remote Control

Abstract. In order to solve the digital home media sharing problems between devices and do some research on UPnP and UPnP AV protocol architecture and then design and implement media sharing system based on embedded intelligent terminal. For smart phones, portable tablet characteristics to achieve a single control point function, support for "push" mode, support for computers, smart phones and other electronic equipment terminal browser capable of all kinds of different platforms as a control point to control the operation. We use eclipse software to development WEB server function module and use dream-weaver software to development the UI. The test results show that the system can not only realize the control exerted on different terminals, but also incorporates UPnP network for remote control technology.

\section{Introduction}

This article discusses how the UPnP technology combined with WEB technology then to interconnect home multimedia resource sharing systems with other devices, and enable remote control system and multi-terminal of the control system.

IDC's digital home by definition refers to the family of media terminal can control and share media resources anytime and anywhere. But the media files on these terminals are independent and it is difficult or impossible to share them among different terminals. This has been a lot of literature have made a wide variety of research. For example, the document 1 on the introduction of an embedded based on the Linux platform UPnP AV (universal plug and play) architecture middleware technology, this technology is only in the embedded platform to achieve control of the system and document 2 on Android gives developers a set of intermediate control system is controlled in this way did the control point to migrate to intelligent terminals, but this is only limited to the terminal and the smart terminal Android, this can not be controlled remotely, the document 3 given by XMPP protocol will be in different locations multimedia integrate resources and achieve specific programs the remote control. The present paper is a combination of various ideas and solutions, and it put forward by the above-mentioned documents presented no platform restrictions, and enables remote control of multimedia control system.

\section{The Related Technology}

UPnP.UPnP technology is a commitment to solve the interconnection between the digital home devices peer network architecture proposed by Microsoft. The architecture work with the Internet and take advantage of the Internet components including IP, TCP, UDP, HTTP, and XML. It uses a 
common agreement to replace the device driver to support interoperability between devices in a scalable network environment. UPnP workflow include: addressing, equipment or use DHCP service-based Auto-IP protocol to obtain an IP address; discovery, discovery of other devices on the home network through SS-DP (simple service discovery protocol) protocol; description, the control point through URL acquisition device and the device contains a detailed description of the service; control, follow the SOAP (simple object access protocol) protocol standards, in accordance with the description information request message transmission operation, complete control; equipment subscribe to event notifications when an event, service status changes; display equipment services and services provided by the state, provides programmable control interface.

Architecture Of AV UPnP.UPnP AV framework defines three separate UPnP components: $\mathrm{CP}$ (control point), MS (media server), MR (media render). Three kinds of components can be any combination within a UPnP device. Its frame is shown below in Figure 1.

MS is shareable storage capacity of the equipment used to publish media resources on the device, such as a NAS (network attached storage). MS offers directory services, connectivity management services and AV transport service (optional).

MR is shareable media decoding capabilities of the device used to play on MS shared media network resources, such as set-top boxes. MR supports playback control services, directory services, and AV content

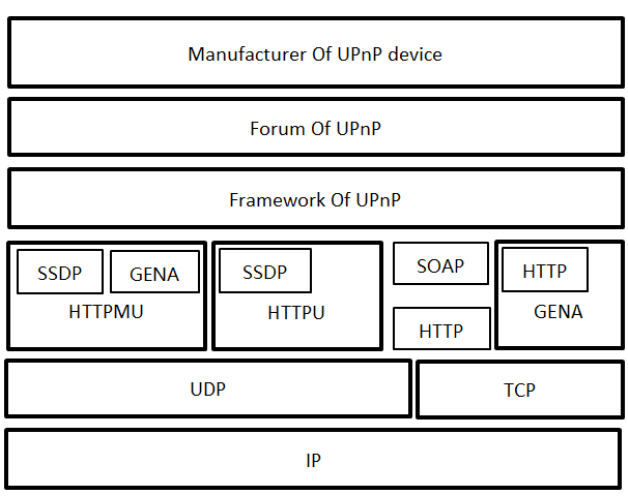

Figure 1 Frame of UPnP delivery service (optional). CP, MS and MR cooperative control work, such as microcomputers. Its main operations include: Detection AV equipment; locate content; acquiring MR supported protocol and format; compare match protocol and format; configure MS / MR; selection; starting content delivery; adjust the studio property; select the next content; clear MS / MR. The architecture has been widely accepted as home networking equipment interoperability accepted standards. The architecture does not perform direct interaction between MS and MR, all coordination $\mathrm{UPnP}$ devices are configured via the $\mathrm{CP}$, but the media

stream-of-band communication protocol to transfer without passing through the $\mathrm{CP}$, is not the case under $\mathrm{CP}$, delivery service can proceed.

Web Server.Web is a typical distributed operating structure, the junction with the exchange of information must involve every client and server. Therefore, Web development technology in general can also be divided into client and server-side technologies technology two categories.

The main task is to show the Web client information content. Web Client design techniques include: HTML language, Java Applets, scripts, CSS, HTML, VRML plug-in technology and technique. Similar technology from a static to a dynamic evolution of the Web client, Web server development technology also evolved from static to dynamic, perfected. Web server technologies including servers, CGI, PHP, ASP, ASP.NET, Servlet and JSP technology.

Because UPnP technology has nothing to do network of this character, so this design can be said Web client server technology any one, considering the portability server, in the present paper uses j2ee technology back-office services, combined XML and AJAX and other common network technology to the entire server part of the system design.

\section{System Design and Implementation}

In the UPnP home network, the device sends SSDP-NOTIFY message to the LAN multicast service itself and its own offer, and control the location of points can be divided into the following two cases.

One.The control point in the case of the internal LAN. In this case you can now send messages directly to 
M-SEARCH request service and are interested in the machine, first looks after the receipt of the message service itself can provide, if they can provide service to reply HTTP $2000 \mathrm{~K}$; if not able to provide the required services equipment, nothing is done.

Two. The control point is not within the LAN. At this point you will need to access the internal LAN network members through Internet, which needs to know the address of the gateway WAN side, that is the gateway IP address obtained dynamically join a network, network address translation, through the firewall, and finally to the internal LAN equipment room to operate.

Based on the study of existing remote access UPnP network method proposed it is a local area network gateway will be located remote control method combining Internet server with access, the second approach used for this purpose. In order to achieve unity of multi-terminal to obtain information, in part joined the UPnP LAN gateway device collection module, UPnP device information collected is transmitted via a LAN off a remote server, the remote server then the message is processed, and let the terminal through IP or binding domain names,

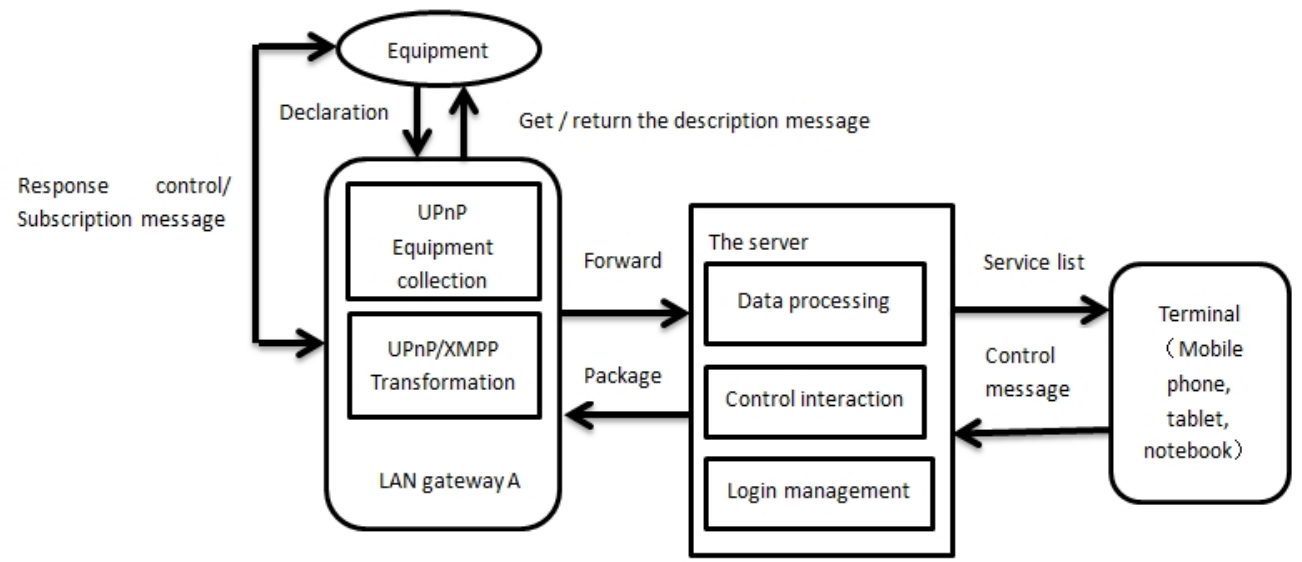

Figure 2 and terminal information returned by the first public network server, and then forwarded to the LAN gateway reply from the LAN gateway to the corresponding controlled device. LAN gateway automatically proxy the operation of the terminal control points and devices within the network request information, and the server implements the remote interactive terminals and UPnP network. Interaction model of the process shown in Figure 2.

Service site design.Server site server technology to build a network. Server system using java program implementation, development on Windows7 system utilizes eclipse software development environment. The Client Access Dreamweaver interface by combining the Firefox browser to development and design. Site identity verification is completed, the work of interactive data processing and forwarding information.

When the user wants to remote control, you first need to log in the server site, then prompts the user to enter a password login page, only after the correct password to follow-up operation, or prompt the user to enter the wrong password. If the user enters the correct password, the trigger LAN gateway server is turned on to receive the message features for home LAN on sending a message to the real-time monitoring, upon receipt of the message, put the received message processing.

Due to the remote control, so the user at a terminal login into the site, but also be able to interact with the site, and this interaction is the control point for remote user control process through the public network server. The whole process has to refresh the service list view media catalog, view services, download services, open the service. Specific processes as shown in Figure 3. 
Since the home network, if the control point within the family network, when they join the home network will apply to the home gateway device IP, home gateway will authenticate the user's identity to ensure information sharing within the LAN device reliability.

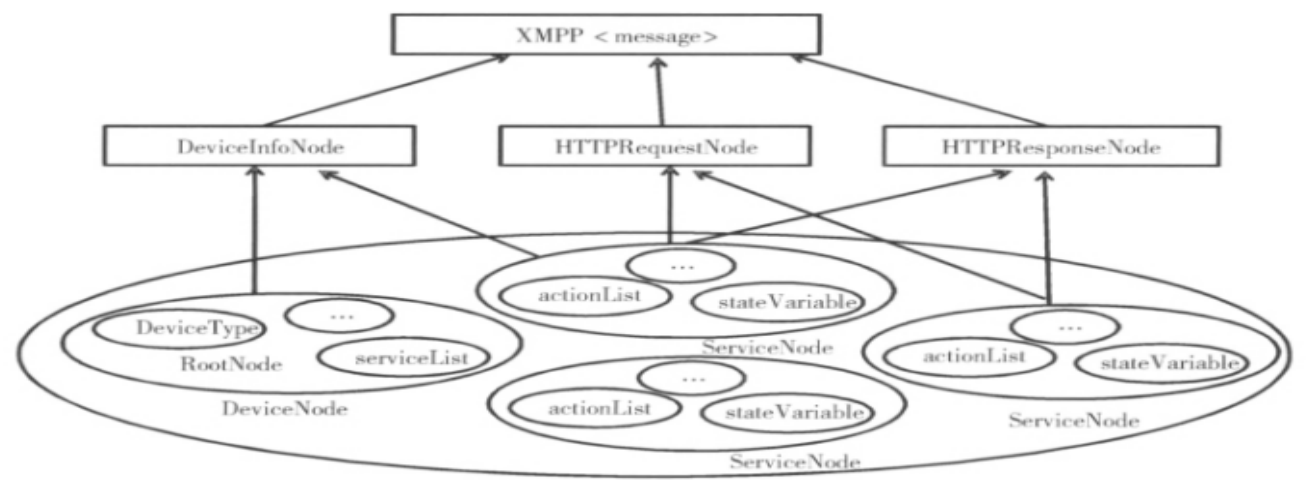

Figure 3 XMPP data package

UPnP equipment collection module. The collection includes a UPnP device supports UPnP protocol stack control points. In the gateway startup process, the control point to search for devices239.255.255.250:1900 address broadcast request, wait qualifying device returns a unicast response message. UPnP device line when the multicast device online announcement, the control point by listening to the same message interface 239.255.255.250:1900 discover UPnP devices and services online publicity. Control Point UPnP device listens to return unicast response message or active release after the online announcement message, initiating obtain device information and service description HTTP request, obtained after a detailed description of the device in response to the document, the corresponding message data package sent to UPnP / XMPP conversion module to the message in the URL Location field.

UPnP device mediation module.After the device receives a remote gateway mediation module transmitted through the server to the UPnP device information, create virtual UPnP device and service descriptions based on the device information, and advertises on the network, in particular, the virtual device and the corresponding remote device share the same device unique logo (uqiue device name, UDN). Return after the virtual device receives local standards issued by the service control point control request information forwarded to the relevant remote gateway at the remote device via $\mathrm{UPnP}$ / XML conversion module and a server, waiting to respond to the remote device to the requesting local control points, for local interactive control points and remote devices.

UPnP/XMPP conversion module.On the one hand, UPnP / XMPP conversion module local UPnP information compression packaged in XMPP message $<$ message $>$ element, transmitted through the server to the terminal; on the other hand, UPnP / XMPP conversion module receives messages from the server forwards the $<$ message $>$ element UPnP restore the contents of the information distributed to other functional modules. XMPP messages are encapsulated and UPnP information recovery include the following three categories:

Equipment information.After receipt of the equipment is passed to the local collection module UPnP device description, UPnP / XMPP conversion module remove one device description and the service description node assembled into new device information node, forwarded to the server. Then save this information data server and automatically creates a corresponding Web page displays information based on the information.

Control request.After logging terminal servers, access to the appropriate device by browsing the site service, once launched UPnP service control request, the request message conversion module and the operation of the device UDN composition encapsulated in HTTP request node XMPP message and forwarded to the remote gateway. Remote Gateway HTTP request after separation of nodes and UDN information fields based on the request message is forwarded to the appropriate remote device, and wait for the device response.

Control responses.Remote Gateway wait for a response to a control device corresponding message, the 
message composition HTTP response node with the device UDN and XMPP message format returned to the originating gateway control request.

Interaction model.Figure 4 shows the terminal server by browsing the site to achieve the entire LAN gateway A remote interaction in the browse and control process. Mainly includes the following steps:

Step 1 Log. Terminal Server by entering the username and password login server, gateway A gateway start time in the automatic logon server.

Step 2 devices collect. Equipment published online proclaimed in the LAN, the gateway A listener to proclaim the message after initiating obtain device information and service description requests to the device. After the device returns the information to the gateway A, the gateway A device information compressed XML data stream encapsulated into XMPP message format, forwarded to the server.

Step 3 The data processing. When the server receives data, the data will be analyzed and processed, and when the terminal access the site and make the appropriate display.

Step 4 control equipment. The terminal may view the device description information displayed on the Site, and to send a message by selecting the control UPnP control device to the server, the server receives a control message, this message will be packaged as XML stream back through server and gateway A forwards it to the LAN A equipment; the device receiving the control message, the same procedure returns control to the server status and dynamic refresh Site.

Step 5 device offline. A local area network when the device is offline, the set Offline declaration prepared release Figure 4

forwarded by post to the server and gateway A package refresh the display.

\section{Summary}

As used here, an open source library

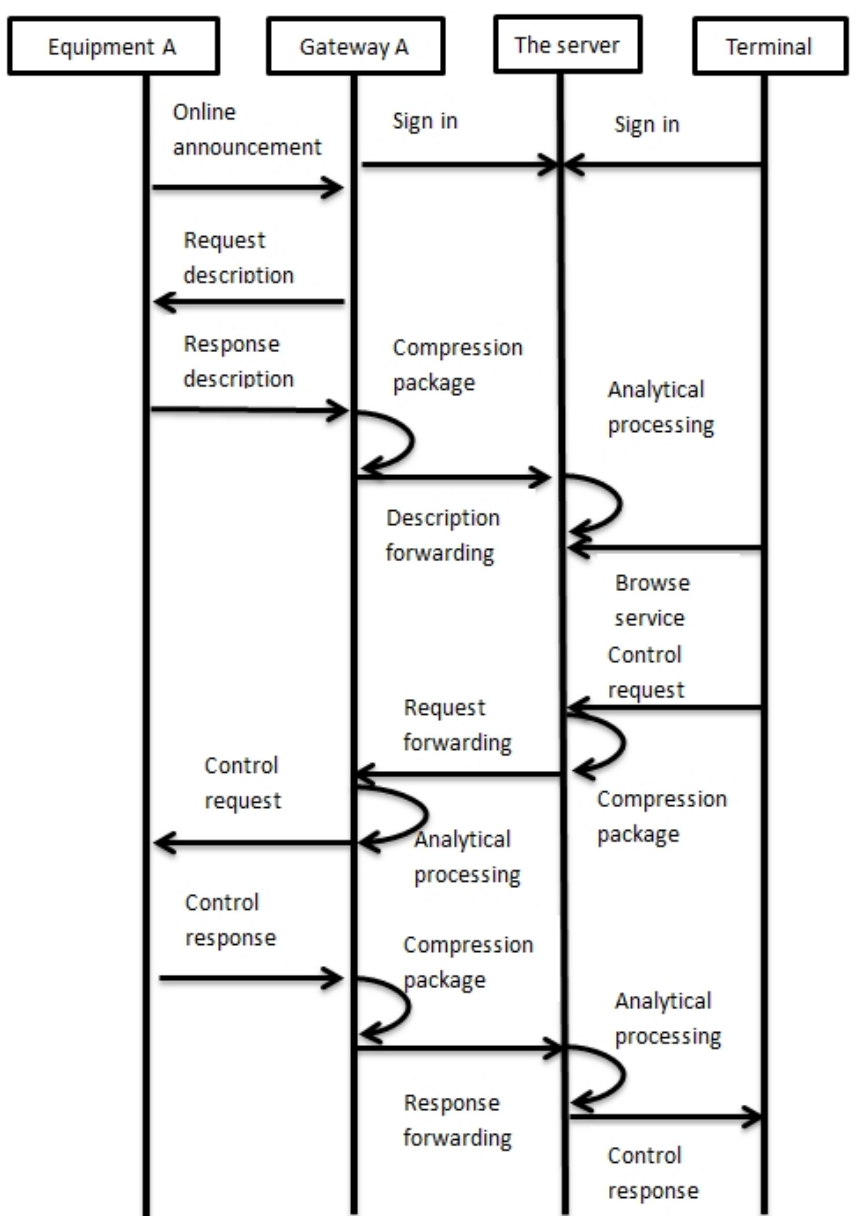
implementation CyberLink UPnP protocol stack, open source library for use Gloox XMPP protocol and OpenFire for XMPP server, the gateway development platform based on Windows operating system and Eclipse integrated development environment. In this paper, on Android, IOS and Windows Mobile PC platform to do the test. Throughout the experiment environment by the gateway, Figure 4 interactive timing diagram server, UPnP device and terminal. Server (IP address 222.197.192. ***) Is located in Internet, gateway A, UPnP device collection module, UPnP / XMPP conversion module located on the LAN A (IP address 10.27.0. ***). Gateway A and the terminal A are able to connect to the server.

After two gateway A server package forwarding, UPnP control point A within the LAN A local UPnP devices can automatically discover A, and the terminal will be able to browse the gateway A UPnP devices and control services online after logging server, and select the appropriate option in the online gateway a operational control in the UPnP device. Thus, the present paper to achieve the successful implementation of LAN UPnP control via remote terminal. By adding certification authority in the server, effectively ensuring the security of network devices. The system uses rich client browser service way to browse and control, embedded linux system different 
from the past or Android client, etc. can be controlled on the development and application of a single client, the successful implementation of intelligent machines, micro-computers, etc. the client has a browser function is controlled, reduce design costs and improve the system efficiency.

\section{References}

[1] Wang Yan. Research on the technology of UPnP equipment control based on embedded [D]. Linux Ocean University of China.2008

[2] Sun Peng, Guo Zhichuan, Sun Xintong, Zhang Wu. Android based media sharing system design and implementation of [J]. computer engineering and design.2014

[3] Zhu Zhenmin, Yang Wei Chen Yuanfei, Hu Yanping. Remote access UPnP network method and implementation. Computer engineering and design [J].2012

[4] Wang Gang, Cheng Gang long, Lin Hao people. [J]. Journal of Wuhan University of Technology.2011 research and implementation of remote collaboration system based on Upnp

[5] Li Fangxing. Research and implementation of multimedia equipment based on UPnP protocol [D].2013

[6] Wu Guoping, Yang Hao, Zheng Xuanming, Ma Li. Streaming media transmission [J]. technology based on UPnP server.2013

[7] Cao sweet. Research and implementation of UPnP media player based on Android system [D]. Nanjing University of Posts and Telecommunications. 2013

The UPnP Forom.UPnP device architecture 1.0.1 [8],

[9].Http://www.upnp.org EB/OL, 2003

Core RFC3920.Extensible messagein and presence protoco. [10] (XMPP) [S]

[11] Petros Belimpasakis, Vlad Stirbu.Remote access to universal plug and play (UPnP) devices utilizing the atom publishing protocol [C].Athens, Greece:Proceedings of the 3rd International Conference on networking and services, 2007; 59-59

[12] Sun Zhihui. Home gateway and terminal remote access design and implementation [D]. Beijing University of Posts and Telecommunications, 2008

[13] Liu Wenzhi, Song Yang Qi.DLNA intelligent home network interoperability to track the [J]. standards and technology, 2009

[13] Zhou Xuefeng. Standard security research for digital home [J]. UPnP technology and information development and economy, 2007

[14] Information on http://www.baidu.com 